\title{
Small-Signal Laplace-Domain Analysis of Uniformly-Sampled Pulse-Width Modulators
}

\author{
David M. Van de Sype*, Koen De Gussemé, Alex P. Van den Bossche, and Jan A. Melkebeek \\ Electrical Energy Laboratory \\ Department of Electrical Energy, Systems and Automation, Ghent University \\ St-Pietersnieuwstraat 41, B-9000 Gent, Belgium \\ *Email: VandeSype@Lambda.UGent.be
}

\begin{abstract}
As the performance of digital signal processors has increased rapidly during the last decade, there is a growing interest to replace the analog controllers in low power switching converters by more complicated and flexible digital control algorithms. Compared to high power converters, the control loop bandwidths for converters in the lower power range are generally much higher. Because of this, the dynamic properties of the uniformly-sampled pulse-width modulators used in low power applications become an important restriction for the maximum achievable bandwidth of control loops. After the discussion of the most commonly used uniformly-sampled pulsewidth modulators, small-signal frequency- and Laplace-domain models for the different types of uniformly-sampled pulse-width modulators are derived theoretically. The results obtained are verified by means of experimental data retrieved from a test setup.
\end{abstract}

\section{INTRODUCTION}

For reasons of price, control circuits for low-power switching power supplies $(<3 \mathrm{~kW})$ are almost always implemented using analog circuits. As the price/performance ratio of digital signal processors has decreased rapidly during the last decade, the interest for digital control of switching power supplies in the low power range has grown [1], [2].

When applying digital control to a switching power supply, the different switches in the supply are often controlled by a digital or uniformly-sampled pulse-width modulator. Consequently, the dynamics of a digitally controlled switching power supply are influenced by two nonlinear effects: quantization effects and modulation effects. As the effects of quantization in digital control of switching power supplies have been addressed before [3], [4], this study focuses on modulation effects. The dynamic behavior of switching power supplies with digital pulse-width modulators in the feedback chain is inherently different from those with analog pulse-width modulators. Hence, the derivation of new models is required. Though exact discrete-time models exist for converters [5], their use in control is limited because of the presence of matrix exponentials and other highly nonlinear vector functions. Approximations of the discrete-time models such as [6], may present usable expressions for control but don't provide the same "feel" for converter and modulator behavior that exists for converters controlled with analog circuitry. To meet these requirements a small-signal Laplace-domain analysis is performed yielding both frequency- and Laplace-domain models for the different types of modulators. The derived models do not only provide important quantitative results but also provide the designer with the necessary insight.

\section{UNIFORMLY-SAMPLED PULSE-WidTH MODUlatorS}

The pulse-width modulators embedded in modern digital signal processors (e.g. TMS320C2XX of Texas Instruments, ADSP2199X of Analog Devices, DSP568XX of Motorola, etc.) operate all in a similar fashion. If we disregard quantization effects, a model for the uniformly-sampled pulsewidth modulator is shown in Fig. 1. The input $u(t)$, a continuous function of time, is sampled with a frequency $\omega_{s}$ synchronously to the pulse-width modulation (Fig. 1). The sampled input $u_{s}(t)$ is sent to a zero-order-hold circuit $(\mathrm{ZOH})$. Finally, the PWM waveform is generated by comparing the output of the $\mathrm{ZOH} u_{H}(t)$ to the value of the carrier waveform $v_{c}(t)$, a triangular waveform. Depending on the shape and the frequency $\omega_{c}$ of the carrier waveform $v_{c}(t)$ different types of uniformly-sampled pulse-width modulators can be obtained. While the carrier frequency $\omega_{c}$ determines the switching frequency, the sampling frequency $\omega_{s}$ defines the number of samples that is taken in a switching period $T_{c}$. In commercial digital controllers two possibilities are commonly offered: the sampling frequency $\omega_{s}$ is equal to the switching frequency $\omega_{c}$ for single-update-mode modulators (Figs. 2 and 3) or the sampling frequency is twice as high as the switching frequency in double-update-mode modulators (Fig. 4). A further subdivision can be made depending on the waveform of the carrier: an isosceles-triangular-carrier or a sawtooth-carrier. When the carrier $v_{c}$ is an isoscelestriangular-carrier waveform, two single-update-mode modulators and one double-update-mode modulator can be identified. The single-update-mode modulators with isosceles-triangularcarrier are the symmetric-on-time modulator Fig. 3(a) and the symmetric-off-time modulator Fig. 3(b). As for the doubleupdate-mode modulators only the modulator with isoscelestriangular-carrier is commercially available, this modulator will be referred to as the double-update-mode modulator Fig. 4. For modulators with a sawtooth carrier only singleupdate-mode modulators are commonly available: the end-ofon-time modulator Fig. 2(a) and the begin-of-on-time modulator Fig. 2(b).

\section{LAPLACE-DOMAIN ANALYSIS}

\section{A. Single-Update-Mode Modulators}

To derive the frequency- and Laplace-domain models of the single-update-mode modulators, the waveforms of the general single-update-mode modulator are used. This general single-update-mode modulator has as carrier waveform $v_{c}$, a triangular waveshape determined by the period $T_{c}$ and the ratio $\alpha$. The latter is the duration of the falling edge of the triangle 


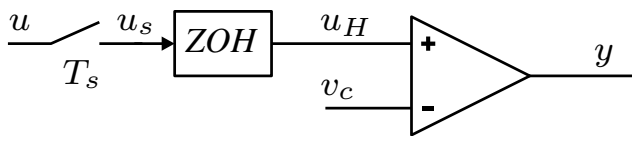

Fig. 1. A general uniformly-sampled pulse-width modulator

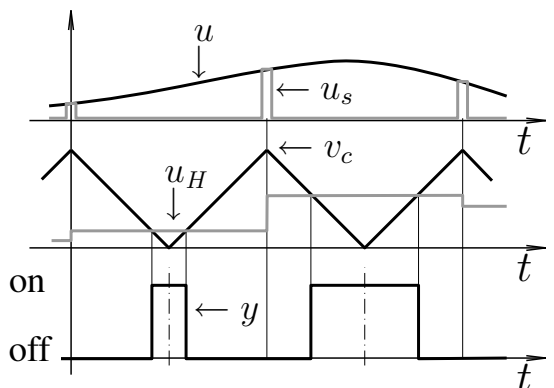

(a)

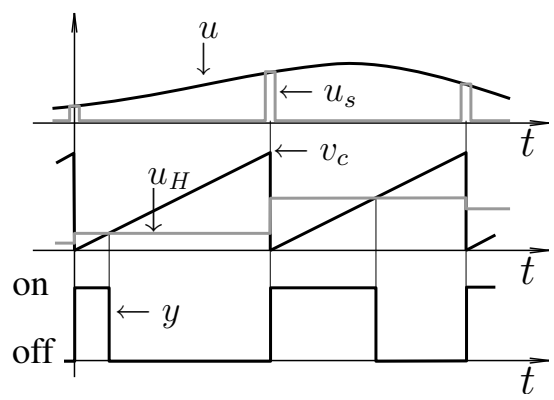

(a)

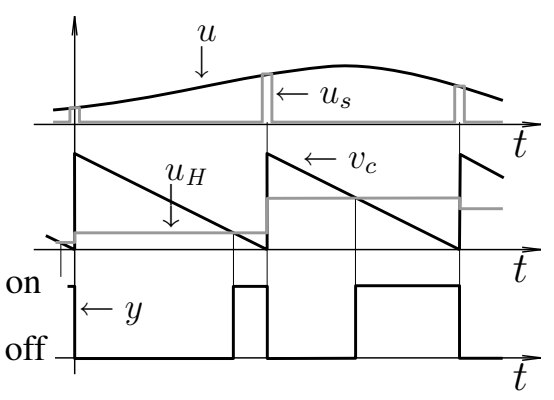

(b)

Fig. 2. The single-update-mode sawtooth-carrier modulators. (a): end-of-on-time, (b): begin-ofon-time

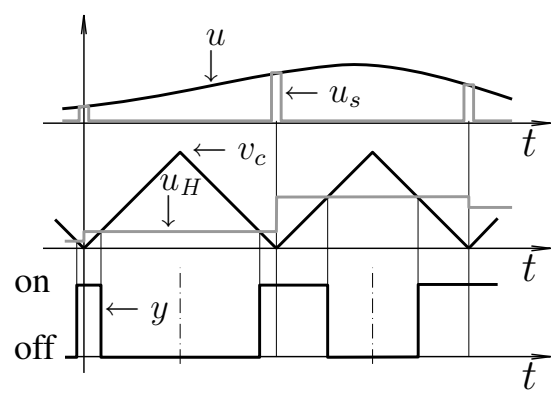

(b)

Fig. 3. The single-update-mode triangular-carrier modulators. (a): symmetric-on-time, (b): symmetricoff-time

relative to the period $T_{c}$ (Fig. 5). Choosing $\alpha$ equal to $0,1 / 2$ and 1 allows to obtain the waveforms for the end-of-on-time modulator, the symmetric-on-time modulator and the begin-

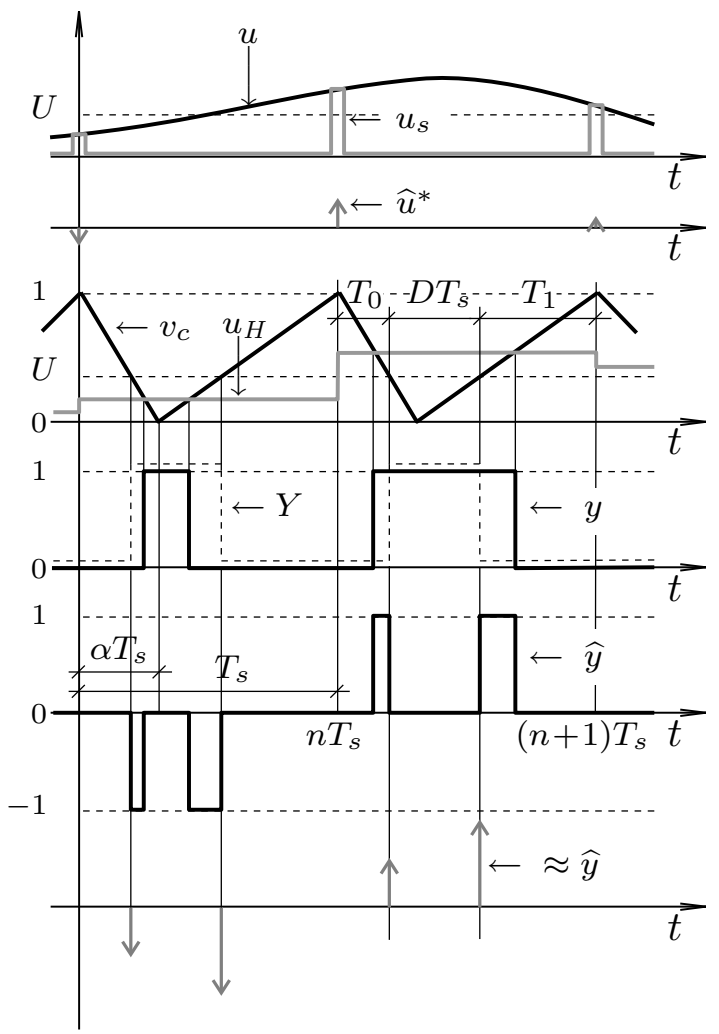

Fig. 5. The key waveforms for a general single-update-mode modulator of-on-time modulator respectively. Hence, three out of four different single-update-mode modulators can be analyzed in a unified way.

The input of the modulator $u(t)$ is separated into a steadystate part $U$ and a small excursion to this steady-state $\widehat{u}(t)$, or

$$
u(t)=U+\widehat{u}(t) .
$$

This input is sampled at the sample rate $T_{s}\left(=T_{c}\right)$ yielding the waveform $u_{s}(t)$. If we use an ideal sampler instead of a real sampler, the small-signal output of the ideal sampler becomes

$$
\widehat{u}^{*}(t)=(u-U)^{*}(t) .
$$

This sampled input is passed on to the $\mathrm{ZOH}$ (see also Fig. 1) before it is compared to the general triangular carrier. The output of the modulator $y(t)$ can also be separated into a steady-state portion $Y(t)$ (the response to $U$ ) and a small excursion to the steady-state $\widehat{y}(t)$, or

$$
y(t)=Y(t)+\widehat{y}(t) .
$$

If the pulses of $\widehat{y}(t)$ are sufficiently small (this can be guaranteed by choosing $\widehat{u}(t)$ sufficiently small), $\widehat{y}(t)$ may be approximated as a series of impulses, in which the separate impulses have the same surface as their corresponding pulses and where the impulses are positioned at the flanks of the steady-state output $Y(t)$ (see Fig. 5, compare the two lower curves).

If we consider the response of the modulator to a smallsignal impulse in the sampled input at time zero

$$
\widehat{u}^{*}(t)=\delta(t),
$$




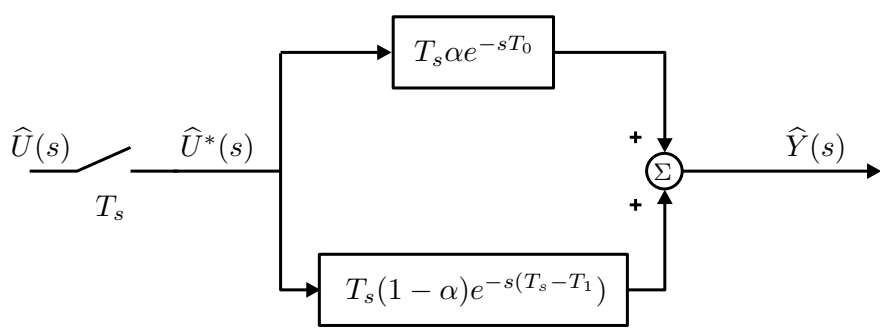

Fig. 6. The block diagram of a idealized general single-update-mode modulator

the small-signal output of the modulator can be approximated by

$$
\widehat{y}(t) \approx T_{s} \alpha \delta\left(t-T_{0}\right)+T_{s}(1-\alpha) \delta\left(t-\left(T_{s}-T_{1}\right)\right),
$$

with $T_{0}$ and $T_{1}$ chosen as in Fig. 5. As the small-signal input is a series of impulses

$$
\widehat{u}^{*}(t)=\sum_{n=-\infty}^{+\infty} \widehat{u}\left(n T_{s}\right) \delta\left(t-n T_{s}\right)
$$

the small-signal output can be written in the Laplace-domain by using (4), (5) and (6)

$$
\widehat{Y}(s)=T_{s}\left(\alpha e^{-s T_{0}}+(1-\alpha) e^{-s\left(T_{s}-T_{1}\right)}\right) \widehat{U}^{*}(s) .
$$

This equation can be schematically represented by the diagram of Fig. 6. (Equation (7) is used in [7] to derive the z-domain models for digitally controlled converters.) Hence, the general single-update-mode modulator behaves, in small signal, as a device that responds to an impulse at its input with two delayed impulses at its output. The impulses at the output are positioned at the switching edges of the steady-state output $Y(t)$ of the modulator (see also Fig. 5).

Taking into account that the Laplace-transform of the sampled small-signal input $\widehat{U}^{*}(s)$ can be expressed as a function of the Laplace-transform of the continuous small-signal input $\widehat{U}(s)[8]$

$$
\widehat{U}^{*}(s)=\frac{1}{T_{s}} \sum_{k=-\infty}^{+\infty} \widehat{U}\left(s-j k \omega_{s}\right)
$$

the small-signal output of the general single-update-mode modulator finally becomes

$\widehat{Y}(s)=\left(\alpha e^{-s T_{0}}+(1-\alpha) e^{-s\left(T_{s}-T_{1}\right)}\right) \sum_{k=-\infty}^{+\infty} \widehat{U}\left(s-j k \omega_{s}\right)$.

Hence, the frequency spectrum of the small-signal output of the modulator contains an infinite number of images of the frequency spectrum of the input with as Nyquist frequency half the sampling frequency, or $\omega_{s} / 2$. If the frequency spectrum of the small-signal input $\widehat{u}(t)$ contains only frequencies lower than the Nyquist frequency and if we consider only frequencies lower that the Nyquist frequency in the output, the latter can be approximated by

$$
\widehat{Y}(s) \approx\left(\alpha e^{-s T_{0}}+(1-\alpha) e^{-s\left(T_{s}-T_{1}\right)}\right) \widehat{U}(s) .
$$

If the proper values for $\alpha, T_{0}$ and $T_{1}$ are chosen, equation (10) provides the frequency- and Laplace-domain models

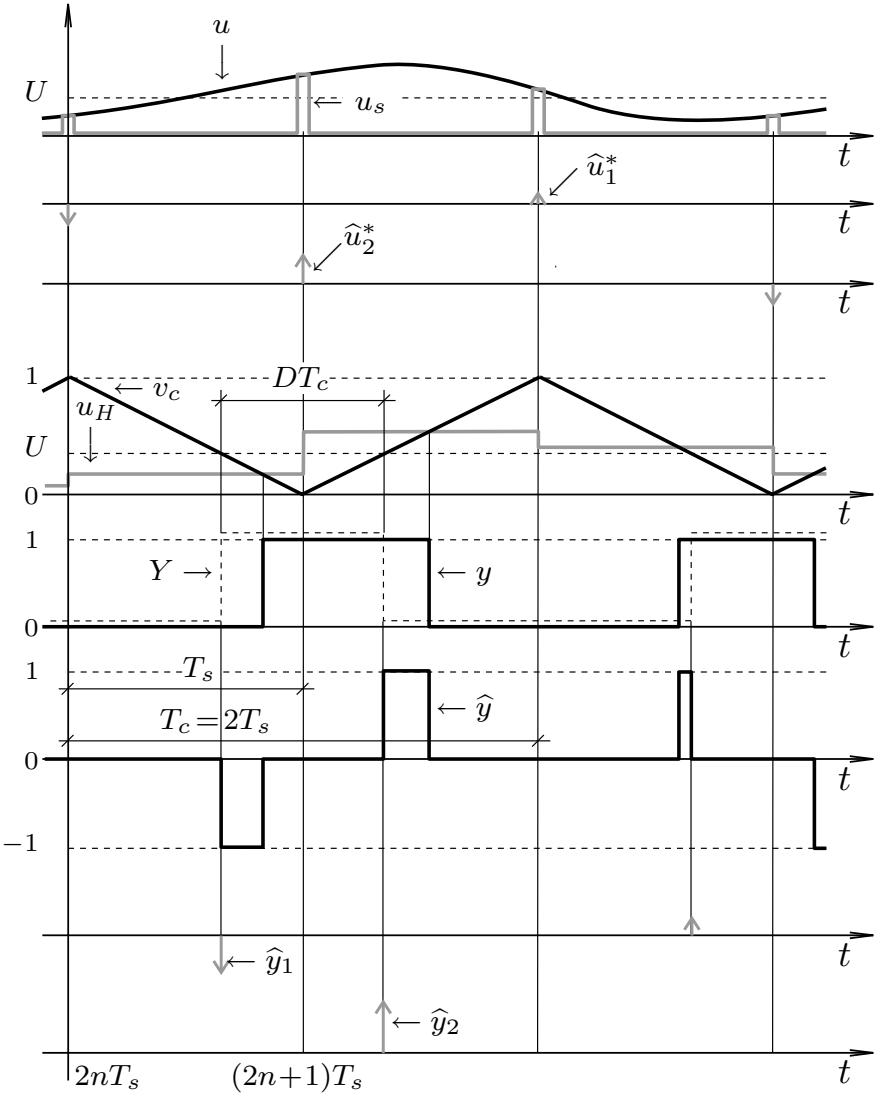

Fig. 7. The key waveforms for the double-update-mode modulator

for the different modulators. For a modulator with a carrier waveform $v_{c}(t)$ limited between 0 and 1 , the average value of the input $U$ is equal to the average duty-ratio $D$. The model for the end-of-on-time modulator is derived from (10) by using $\alpha=0, T_{1}=(1-D) T_{s}$, or

$$
G_{P W M}(s)=\frac{Y(s)}{U(s)}=e^{-s D T_{s}} .
$$

For $\alpha=1, T_{0}=(1-D) T_{s}$ the Laplace-domain model for the begin-of-on-time modulator becomes

$$
G_{P W M}(s)=e^{-s(1-D) T_{s}} .
$$

The model for the symmetric-on-time modulator is obtained by substituting $\alpha=1 / 2$ and $T_{0}=T_{1}=(1-D) / 2$ ) in (10)

$$
G_{P W M}(s)=\frac{1}{2}\left(e^{-s \frac{(1-D) T_{s}}{2}}+e^{-s \frac{(1+D) T_{s}}{2}}\right)
$$

The Laplace-domain model of the symmetric-off-time modulator can't be derived directly from (10). From a similar analysis the following model is obtained

$$
G_{P W M}(s)=\frac{1}{2}\left(e^{-s \frac{D T_{s}}{2}}+e^{-s \frac{(2-D) T_{s}}{2}}\right)
$$

\section{B. The Double-Update-Mode Modulator}

For the double-update-mode modulator the series of impulses $\widehat{u}^{*}(t)(6)$ at the input of the modulator with sampling period $T_{s}$ is divided into two subseries with sampling period 


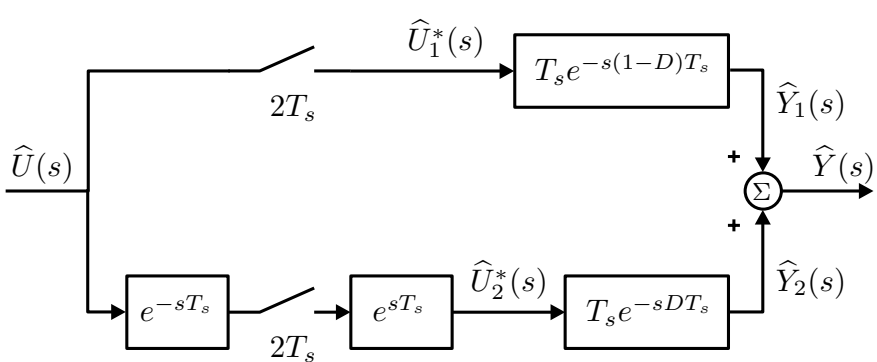

Fig. 8. The block diagram of the idealized double-update-mode modulator

$T_{c}\left(=2 T_{s}\right)$, (see Fig. 7):

$$
\left\{\begin{array}{l}
\widehat{u}_{1}^{*}(t)=\sum_{n=-\infty}^{+\infty} \widehat{u}(t) \delta\left(t-n T_{c}\right) \\
\widehat{u}_{2}^{*}(t)=\sum_{n=-\infty}^{+\infty} \widehat{u}(t) \delta\left(t-n T_{c}-T_{s}\right)
\end{array} .\right.
$$

The response of the modulator to these separate subseries is different. If we consider an impulse in the first subseries at time zero

$$
\widehat{u}_{1}^{*}(t)=\delta(t)
$$

the response of the modulator can be approximated by an impulse (Fig. 7)

$$
\widehat{y}_{1}(t)=T_{s} \delta\left(t-(1-D) T_{s}\right) .
$$

An impulse in the second subseries

$$
\widehat{u}_{2}^{*}(t)=\delta(t)
$$

yields approximately the output

$$
\widehat{y}_{2}(t)=T_{s} \delta\left(t-D T_{s}\right) \text {. }
$$

By considering the above, the output of the double-updatemode modulator can be expressed in the Laplace domain as

$$
\begin{aligned}
\widehat{Y}(s) & =\widehat{Y}_{1}(s)+\widehat{Y}_{2}(s) \\
& =T_{s}\left(e^{-s(1-D) T_{s}} \widehat{U}_{1}^{*}(s)+e^{-s D T_{s}} \widehat{U}_{2}^{*}(s)\right) .
\end{aligned}
$$

Hence, for small signals the dynamic behavior of the doubleupdate-mode modulator can be represented schematically by Fig. 8 .

As both subseries, $\widehat{u}_{1}^{*}(t)$ and $\widehat{u}_{2}^{*}(t)$ (15), are obtained by sampling the same continuous small-signal input $\widehat{u}(t)$, their Laplace transforms are related [8]:

$$
\left\{\begin{array}{l}
\widehat{U}_{1}^{*}(s)=\frac{1}{T_{c}} \sum_{k=-\infty}^{+\infty} \widehat{U}\left(s-j k \omega_{s}\right) \\
\widehat{U}_{2}^{*}(s)=\frac{1}{T_{c}} \sum_{k=-\infty}^{+\infty} e^{-j k \omega_{c} T_{s}} \widehat{U}\left(s-j k \omega_{s}\right)
\end{array} .\right.
$$

By substituting these Laplace transforms in (20), an expression similar to (9) is obtained for the double-update-mode modu- lator:

$$
\begin{aligned}
\widehat{Y}(s)=\frac{1}{2} e^{-s(1-D) T_{s}} \sum_{k=-\infty}^{+\infty} \widehat{U}\left(s-j k \omega_{c}\right) \\
+\frac{1}{2} e^{-s D T_{s}} \sum_{k=-\infty}^{+\infty}(-1)^{k} \widehat{U}\left(s-j k \omega_{c}\right)
\end{aligned}
$$

Note that, though the sampling frequency for the doubleupdate modulator is twice as high as the switching frequency, the Nyquist frequency for this modulator is half the switching frequency $\omega_{c} / 2$.

Under the assumption that the frequency spectrum of the input $\widehat{u}(t)$ contains only frequencies below the Nyquist frequency, and that we are only interested in the frequency content of the output in the same frequency range, the smallsignal output of the double-update-mode modulator can be approximated by

$$
G_{P W M}(s) \approx \frac{1}{2}\left(e^{-s(1-D) T_{s}}+e^{-s D T_{s}}\right) .
$$

\section{Discussion of Results}

The Laplace-domain models for the single-update-mode and double-update-mode modulators are summarized in Table I. To derive the corresponding frequency-domain models, the Laplace operator $s$ is replaced by $j \omega$. The resulting frequencydomain models are also shown in Table I.

Whereas the models for the end-of-on-time modulator have been published before and are in agreement with the results of [9], [10] and [11], the models for the other modulators are new expressions. The frequency-domain models for the sawtooth-carrier modulators (end-of-on-time and begin-of-ontime, Table I) show that these modulators behave as a pure delay that is dependent on the average duty-ratio $D$. This delay can be regarded upon as caused by the delay between the taking of the sample and the response of the modulator to this sample: either $D T_{s}$ for the end-of-on-time modulator or $(1-D) T_{s}$ for the begin-of-on-time modulator $\left(T_{s}=T_{c}\right)$.

Conversely, the single-update-mode isosceles-triangularcarrier modulators (symmetric-on-time, symmetric-off-time, Table I) have a gain that is dependent on the frequency and the average duty-ratio $D$, while the phase-shift represents a delay of half a sampling-period. The delay of $T_{s} / 2$ can be intuitively understood. After all, the response $\widehat{y}(t)$ of a symmetric modulator to a new sample at the input consists of two parts, a part before and a part past half of the switching period (see Fig. 5 for $\alpha=\frac{1}{2}$ ). Hence, on average, the response to a sample occurs in the center between the two parts or with a delay of half a switching period.

The frequency domain model for the double-update-mode (Table I) shows again a dependency on the average duty-ratio $D$ and the frequency of the input $\omega$. Moreover, only the gain varies with the average duty-ratio $D$, while the delay is fixed at half a sampling frequency $T_{s} / 2$. This delay can be explained as follows. The waveform of the double-update-mode modulator (Fig. 7) can also be constructed by applying one sample to a begin-of-on-time modulator and the other to a end-of-on-time modulator. As a consequence, the delay to the samples $\widehat{u}_{1}(t)$ 
TABLE I

THE FREQUENCY- AND LAPLACE-DOMAIN MODELS FOR UNIFORMLY-SAMPLED PULSE-WIDTH MODULATORS

\begin{tabular}{|l|c|c|}
\hline & $G_{P W M}(s)$ & $G_{P W M}(j \omega)$ \\
\hline end-of-on-time & $e^{-s D T_{s}}$ & $1 \angle\left(-j \omega D T_{s}\right)$ \\
\hline begin-of-on-time & $e^{-s(1-D) T_{s}}$ & $1 \angle\left(-j \omega(1-D) T_{s}\right)$ \\
\hline symmetric-on-time & $\frac{1}{2}\left(e^{-s \frac{(1-D) T_{s}}{2}}+e^{-s \frac{(1+D) T_{s}}{2}}\right)$ & $\cos \left(\frac{\omega D T_{s}}{2}\right) \angle\left(-\frac{\omega T_{s}}{2}\right)$ \\
\hline symmetric-off-time & $\frac{1}{2}\left(e^{-s \frac{D T_{s}}{2}}+e^{-s \frac{(2-D) T_{s}}{2}}\right)$ & $\cos \left(\frac{\omega(1-D) T_{s}}{2}\right) \angle\left(-\frac{\omega T_{s}}{2}\right)$ \\
\hline \hline double-update-mode & $\frac{1}{2}\left(e^{-s(1-D) T_{s}}+e^{-s D T_{s}}\right)$ & $\cos \left(\omega\left(D-\frac{1}{2}\right) T_{s}\right) \angle\left(-\frac{\omega T_{s}}{2}\right)$ \\
\hline
\end{tabular}

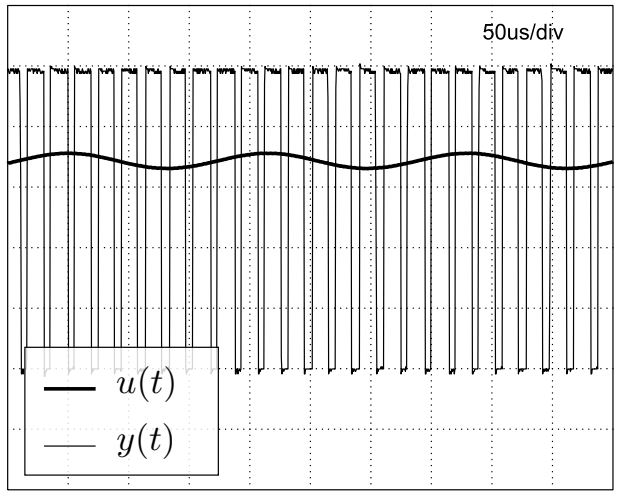

Fig. 9. The experimentally measured in- and output of an end-of-on-time modulator

is $(1-D) T_{s}$, while the delay to the samples $\widehat{u}_{2}(t)$ is $D T_{s}$, or on average $T_{s} / 2$.

Note that the Nyquist frequency for both single-updatemode and double-update-mode modulators is equal to half the switching frequency $T_{c} / 2$ (for single-update-mode modulators $T_{s}=T_{c}$ ). Hence, if these models are used to predict the closedloop stability of a digitally controlled converter, their validity is limited to frequencies below half the switching frequency.

\section{EXPERIMENTAL RESULTS}

Experimental verification of the theoretical results obtained was performed using the digital pulse-width modulators of the ADMC401 of Analog Devices. For this purpose a nonsynchronous sine wave with a small amplitude, programmable frequency and offset is supplied to the analogue-to-digital converter (ADC) of the DSP (Fig. 9); the ADC is synchronized with the PWM. The sample obtained by the ADC is passed on to the pulse-width modulator. Both the signal at the input of the ADC and the signal at the output of the modulator are provided to two matched first order low pass filters (with a time constant of $33 \mu \mathrm{s}$ ) before they are offered to the inputs of a digital oscilloscope. The attenuation and phase shift due to the low pass filtering is the same for both signals. As the applied sine wave is non-synchronous, the averaging function of the oscilloscope allows to clearly visualize the fundamental

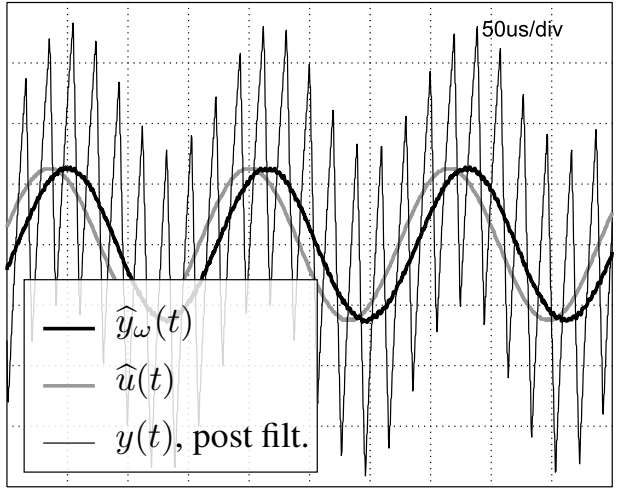

Fig. 10. The small-signal input $\widehat{u}(t)$ and output $\widehat{y}_{\omega}(t)$ after filtering and averaging, together with the filtered output $y(t)$ for the end-of-on-time modulator.

$y_{\omega}(t)$ of the PWM output (Fig. 10). The experimental results in this section are all obtained by comparing the amplitude and phase shift of the two signals on the oscilloscope.

The switching frequency $\omega_{c} / 2 \pi$ of the modulator was set to $51 \mathrm{kHz}$, corresponding to a switching period $T_{c}$ of $19.6 \mu \mathrm{s}$. Fig. 11 shows the theoretical delay introduced by an end-ofon-time modulator as a solid line; experimental results are added for comparison. As the gain of this modulator is always unity, no plot is added, nevertheless a good correspondence to experiments was noticed. A similar experiment was performed to check the validity of the transfer function for the begin-ofon-time modulator, the results are exhibited in Fig. 12. The comparison between theoretical results and the experimental measurements show the validity of the approach.

As for the isosceles-triangular-carrier modulators only the gain of the frequency-domain model is variable with $D$, phase plots have been omitted. The gain for the symmetric-on-time modulator, the symmetric-off-time and the double-updatemode modulator are shown in Fig. 13, Fig. 14 and Fig. 15, respectively. Note the subtle difference between Figs. 13 and 14: in Fig. 13 the lowest curve corresponds to $D=0.95$ while in Fig. 14 this curve matches $D=0.05$. Again, a good correspondence exists between the experimental data and the theoretical results. 


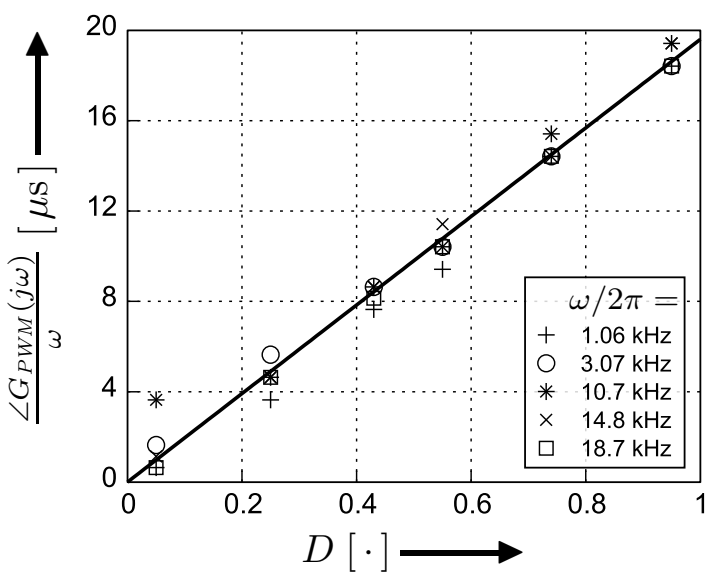

Fig. 11. Experimental measurement of the transport delay of the end-ofon-time modulator as a function of the input frequency $\omega$ and the average duty-ratio $D$

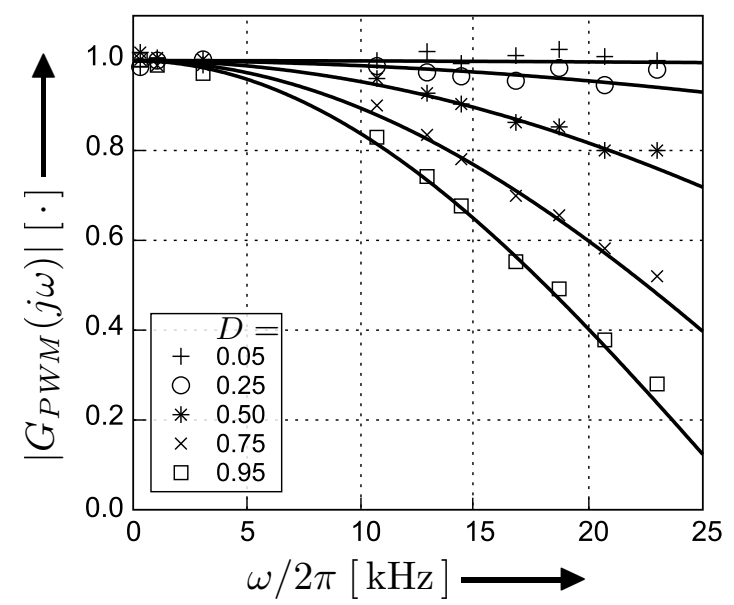

Fig. 13. Experimental measurement of the gain of the symmetric-on-time modulator as a function of the input frequency $\omega$ and the average duty-ratio $D$

\section{CONClusion}

The increasing performance, the good flexibility and the decreasing price of digital control circuitry have entailed a renewed interest in the digital control of switching power supplies. A commonly encountered part of such a digital control circuit is the uniformly-sampled pulse-width modulator. In modern microcontrollers different types of digital pulsewidth modulators can be categorized, depending on the ratio between the sampling-frequency and the switching-frequency, and the shape of the carrier waveform. Four types of singleupdate-mode modulator and one type of double-update-mode modulator can be distinguished. The single-update-mode modulators are the symmetric-on-time modulator, symmetric-offtime modulator, the end-of-on-time modulator and the beginof-on-time modulator. As there is in most cases only one pulsewidth modulator using a double update mode, it is simply called the double-update-mode modulator.

Of the 5 different types of these pulse-width modulators dynamic models only exist for the end-of-on-time modulator. To fill this void the small-signal Laplace-domain analysis for the different types of uniformly-sampled pulse-width modula-

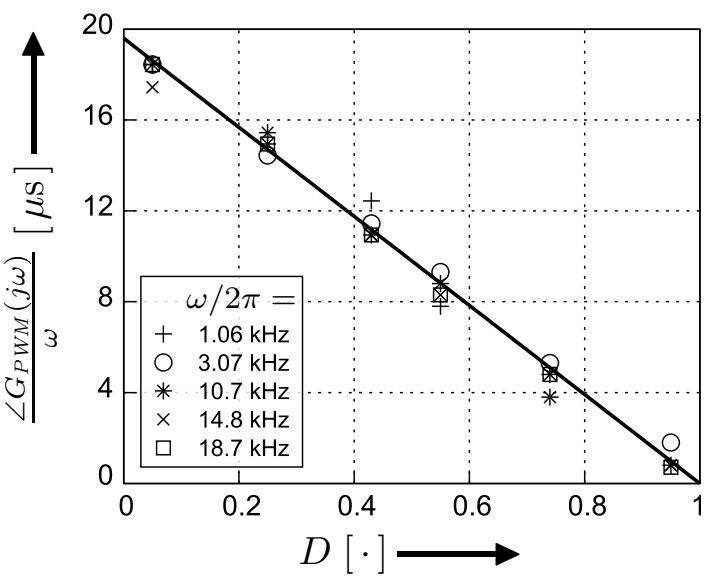

Fig. 12. Experimental measurement of the transport delay of the begin-ofon-time modulator as a function of the input frequency $\omega$ and the average duty-ratio $D$

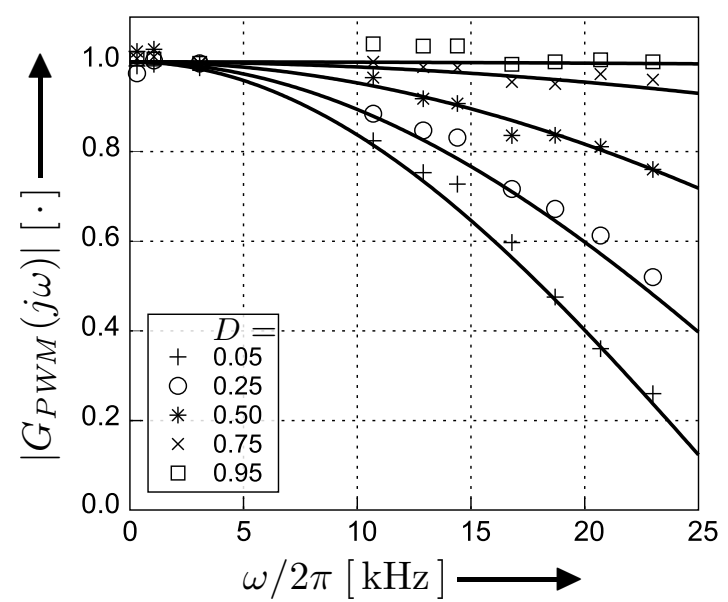

Fig. 14. Experimental measurement of the gain of the symmetric-off-time modulator as a function of the input frequency $\omega$ and the average duty-ratio D

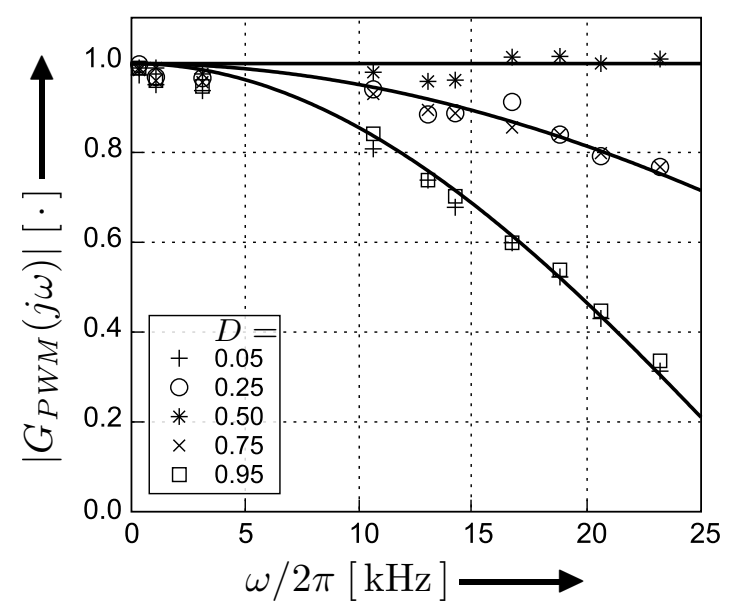

Fig. 15. Experimental measurement of the gain of the double-update-mode modulator as a function of the input frequency $\omega$ and the average duty-ratio

tors is performed. As a result of this analysis both frequencydomain models and Laplace-domain models for the different modulators are derived. The general conclusions are that 
modulators with a sawtooth carrier behave dynamically as a pure transportation delay dependent on the average value of the duty-ratio, while the isosceles-triangular-carrier modulators have a fixed delay of half a sampling period with a gain that varies with the applied frequency and the average value of the duty-ratio. Moreover, for both single-update-mode and double-update-mode modulators the Nyquist frequency is half the switching frequency. The obtained models are verified by experiment using the pulse-width modulators on-board the ADMC401 of Analog Devices.

\section{REFERENCES}

[1] — , "Power supply 'Digital Control'-Real or Virtual," RAP session, 18th Ann. Appl. Power Electron. Conf. and Exp. (APEC2003), Miami Beach, USA, Feb. 9-13, 2003.

[2] - , "Special issue on digital control in power electronics," IEEE Trans. Power Electron., Vol. 18, No. 1, Part II of Two Parts, Jan. 2003.

[3] D.A. Grant, M. Stevens, and J.A. Houldsworth, "The effect of word length on the harmonic content of microprocessor-based PWM waveform generators," IEEE Trans. Ind. Applic., Vol. 21, No. 1, Jan./Feb. 1985, pp. 218-225.
[4] A.V. Peterchev, S.R. Sanders, and J.A. Houldsworth, "Quantization resolution and limit cycling in digitally controlled PWM converters," Proceedings IEEE 32nd Annual Power Electr. Spec. Conf., Vancouver, Canada, 2001, on cd-rom.

[5] G.C. Vergehese, M.E. Elbuluk, and J.G. Kassakian, “ A general approach to sampled-data modeling for power electronic circuits," IEEE Trans. Power Electron., Vol. 1, Apr. 1986, pp. 76-89.

[6] V. Rajasekaran, J. Sun, and B.S. Heck, "Bilinear discrete-time modeling for enhanced stability prediction and digital control design," Trans. Power Electron., Vol. 18, No. 1, Jan. 2003, pp. 381-389.

[7] D.M. Van de Sype, K. De Gussemé, A.P. Van den Bossche, J.A. Melkebeek, "Small-signal z-domain analysis of digitally controlled converters," Proc. of the 35th Ann. Power Electron. Spec. Conf., Aachen, Germany, 2004 , on cd-rom.

[8] G.F. Franklin, J.D. Powell, and M. Workman, "Digital control of dynamic systems," Addison Wesley Longman, California, USA, 3rd edition, 1998.

[9] G.W. Wester, and R.D. Middlebrook, "Low-frequency characterization of switched dc-dc converters," IEEE Trans. Aerosp. and Electr. Syst., Vol. 9, No. 3, May 1973, pp. 376-385.

[10] D.M. Mitchell, "Pulsewidth modulator phase-shift," IEEE Trans. Aerosp. and Electr. Syst., Vol. 16, No. 3, May 1980, pp. 272-278.

[11] R. Tymerski, "Frequency analysis of time-interval-modulated switched networks," Trans. Power Electron., Vol. 6, No. 2, Apr. 1991, pp. 287 295. 